\title{
All parts of an item are not equal: Effects of phonological redundancy on immediate recall
}

\author{
ELISABET SERVICE and SINI MAURY \\ University of Helsinki, Helsinki, Finland
}

\begin{abstract}
The process of redintegration is thought to use top-down knowledge to repair partly damaged memory traces. We explored redintegration in the immediate recall of lists from a limited pool of partly phonologically redundant pseudowords. In Experiment 1, four kinds of stimuli were created by adding the syllable /ne/to two-syllablepseudowords, either to the middle (/tepa/vs. /tenepa/) or to the end (/tepane/), or adding a different syllable to each item (/tepalo/, /vuropi/). The repeated syllable was thought to be available for redintegration. Lists of two-syllable pseudowords were recalled best, items with a redundant end were intermediate, and items with a redundant middle-syllable were as hard as nonredundant three-syllableitems. In Experiment 2, the last syllable was predictable from context but not shared between all stimuli, reducing phonological similarity between items. Performance did not differ from the situation with identical last syllables. In Experiment 3, a shared first syllable had a detrimental effect on memory. An error analysis showed that beneficial redundancy effects were accompanied by harmful similarity effects, impairing memory for nonredundant syllables. The balance between the two effects depended on syllable position.
\end{abstract}

New findings are continually refining our understanding of the functioning of the phonological loop. This is the component of working memory that is responsible for immediate recall of sequences of verbal items in the working memory framework of Baddeley and Hitch (1974; Baddeley, 1986). An increasing body of data has emerged lately that addresses the precise mechanisms of top-down influences in immediate serial recall. Specifically, a number of studies have found evidence for reconstructive processes, so-called redintegration, that operate to restore incomplete phonological traces for output (Gathercole, Frankish, Pickering, \& Peaker, 1999; Gathercole, Pickering, Hall, \& Peaker, 2001; Hulme, Maughan, \& Brown, 1991; SaintAubin \& Poirier, 2000; Schweickert, 1993). Initially these processes were described as related to stored lexical representations, explaining why words are easier to recall than pseudowords (Hulme et al., 1991; Saint-Aubin \& Poirier, 2000).

Neuropsychological evidence for word-level top-down influences on serial recall comes from a study of a patient with naming difficulties (Knott, Patterson, \& Hodges, 2000). She had a significantly reduced ability to use semantic entries to activate lexical form representations, resulting in a pronounced anomia. At the same time, her ability to repeat word sequences was impaired by numer-

Some of the data were first presented at the 40th meeting of the Psychonomic Society in Los Angeles, November 1999. The research was funded by the Academy of Finland, Grant 39253. We thank Susan Gathercole and two anonymous reviewers for thoughtfulcomments on a previous version of the manuscript. Correspondence concerning this article should be addressed to E. Service, Department of Psychology and Neuroscience, Life Sciences Centre, Dalhousie University, Halifax, NS B3H 4J1, Canada (e-mail: eservice@dal.ca). ous phonologicalerrors. What was interesting was that her immediate serial recall for the words that she had been able to produce in a picture naming task was clearly better than her performance on other words that had not been available to her in the naming task. This pattern suggested that the word forms that were unavailable to her in speech production caused her the kind of phonologicaldifficulties nonwords tend to do for normal subjects. This led the authors to conclude that immediate serial recall of the phonological forms of items is highly dependent on lexical representations being available to the speech planning system. It has generally been assumed that these representations are used at the time of overt recall, but it is also conceivable that they are used during rehearsal. The present study investigated how top-down information can affect the immediate recall of phonologicalforms. Specifically, we explored the relationship between the helpful effect of phonological predictability and the harmful effect of phonological confusability at the syllable level on recall.

Not only words, but also pseudowords, can be affected by top-down clean-up processes in immediate recall. The language-specific phonotactic probabilities of the phoneme transitions that occur in a pseudoword affect the likelihood that the pseudoword is recalled in its correct form (Gathercole et al., 1999). In recall of sequences of consonant-vowel-consonant (CVC) pseudowords, the majority of errors were blends between different items in the sequence, resulting from a phoneme in a different item in the sequence migrating to a corresponding position in the incorrectly recalled item (Gathercole et al., 2001). Another frequent error category comprised incorrectly recalled items that included phonemes not occurring in the same position in any item on the list. Blends were especially common in phonologically similar lists, 
sharing the vowel. Order errors at the level of whole items were rare (1\% in phonologically distinct and $8 \%$ in similar lists). Such results could be explained by assuming that the other items in a list of nonwords compete with the item to be output. The less that discriminating phonological information is available for the item, the more likely it is that it is affected by some other item on the list (or an item in a limited stimulus pool). In the study by Gathercole and her colleagues, vowels were recalled better than consonants. When this distinctive information was unavailable in the phonologically similar lists, there was a $52 \%$ increase in errors due to consonant migrations from other items.

The studies by Gathercole and her colleagues used a design in which each stimulus item was presented only once to each participant. Thus, the top-down influences for both words and pseudowords in their studies must have been based on previously existing vocabulary in long-term memory. In our study, we focused on another basis for redintegration-learning occurring in connection with the experiment when the stimulus pool of pseudowords is limited and participants are allowed to familiarize themselves with the stimuli before the beginning of the experiment. Another difference between our study and those by Gathercole et al. is that they used monosyllabic stimuli, whereas we studied three-syllable pseudowords in an attempt to see how memory for different syllable positions might be affected by phonological redundancy. Redundancy was operationalized as shared $\mathrm{CV}$-syllables between list items.

In addition to the wish to study the mechanisms of redintegration in general, the present study was also motivated by the need to understand possible top-down influences in a previous set of experiments. The most widely accepted explanation for the well-documented advantage in immediate recall for short words relative to long words is that the phonological loop is time limited. Therefore, more words of short articulatory duration than words of long duration can be rehearsed in the time window that supports full phonological representations before these decay (Baddeley, Thomson, \& Buchanan, 1975). A finding in some previous experiments (Service, 1998) supported an alternative explanation - that such word-length effects could arise from phonological complexity (see Caplan, Rochon, \& Waters, 1992; Caplan \& Waters, 1994; Nairne, 1990). Since these results were based on memory for pseudowords, the present study also asked whether top-down influences at work with pseudoword stimuli could undermine the suggested phonologicalcomplexity explanation and thus indirectly support the better established duration explanation.

A study by Service (1998) took advantage of a feature of the Finnish language - that the lengths of phoneme sounds are used to distinguish between words- to construct two sets of pseudoword stimuli that were matched for length of articulatory duration but differed in number of phonemes. One set was made up from short-duration CVCV items (e.g., /tepa/) by lengthening one of the vowel and one of the consonant sounds (e.g., /te:pra/,/tepra:/). The other set was constructed from the short items by adding a syllable and then changing the first syllables so that the original short item did not occur as such in the stimuli (e.g., /tepavi/ $\rightarrow$ /ropavi/). The two-syllable set with the long speech sounds took equally long to pronounce as the set with three syllables. According to the duration hypothesis, more short-duration two-syllable pseudowords should have been remembered than long-duration pseudowords. There should have been no difference between the two kinds of long-duration items. This was not what was found. On the contrary, there was no statistically reliable difference (or only a marginal one in a second experiment) between the short- and the long-duration items with four phoneme sounds, whereas the six-phoneme items were clearly more difficult to remember. The results suggested that the number of phoneme transitions was a major factor contributing to the item-length effect. However, the lack of an item-length effect between the four-phoneme items of different durations could have resulted from beneficial top-down influences on the long stimuli, compensating for time-related decay. This could have happened because the longer variants had a redundant structure (always a long vowel and a long consonant), which could have helped to support successful repair of degraded representations. In addition to exploring the character of phonological top-down effects on the recall of nonwords in general, this study also investigated the hypothesis that the failure to detect an item-length effect in the previous study could have resulted from redintegrative processes based on phonological learning during the experiments.

The present set of experiments explored top-down effects on three-syllable CVCVCV pseudowords of the kind that were used in the earlier Finnish experiments, investigating the effects of phonological complexity and item duration in immediate recall. We compared memory for short CVCV stimuli with memory for three-syllable stimuli that had a nonredundant structure - that is, variable consonants and vowels in all positions and threesyllable stimuli that shared syllables. Since one aim was to find out the extent to which such phonological redundancy could help memory, the first experiment included stimuli that we thought would be most helpful—stimuli that shared either the middle or the last syllable. A condition with a shared first syllable was not included since we thought it would lead to phonological confusion between items as the shared vowel had done in Gathercole et al.'s lists. A study using the false memory paradigm has also provided some evidence that the initial two phonemes of CVC words have a special status. They played a prominent role in predicting false memories for unpresented items with this structure (Westbury, Buchanan, \& Brown, 2002). In our experiments, we asked whether phonological redundancy in three-syllable pseudowords could obliterate the recall advantage for short two-syllable stimuli and whether this depended on the syllabic position of the redundancy. A positive answer would cast doubt over the phonological complexity interpretation of the earlier results. 


\section{EXPERIMENT 1}

\section{Method}

Participants. Fifteen participants (mean age $=24.5$ years, $S D=$ 4.4), 12 females and 3 males, took part in the study as volunteers or for course credit. All participants spoke Finnish as their native language, and none had had any known problems with learning to read or spell.

Stimuli. There were four pools of 12 stimuli each. The first pool consisted of short two-syllable CVCV (e.g., /kula/, /jalu/) pseudowords similar to those used in Service (1998). The second pool of long-middle-redundant pseudowords was made by adding the syllable /ne/ between the first and second syllables of the short stimuli (e.g., /kunela/, /janelu/). The long-end-redundant stimuli were constructed by adding the syllable /ne/ to the ends of the short pseudowords (e.g., /kulane/, /jalune/). These stimuli sounded as if they rhymed with each other. Finally, a long-nonredundant pool of stimuli was created by adding unpredictable CV syllables to the ends of the short stimuli. These stimuli resembled the three-syllable stimuli in the previous study. Each of the four pools of items was used to construct lists of growing length, starting at List Length 3 to List Length 9. There were 10 lists of each length for each of the four types of item.

Procedure and Design. The participants were allowed to study the four alphabetized lists of the stimuli that were used in the experiment. They were then asked to read the stimuli aloud at as fast a pace as they felt comfortable with without losing accuracy. Their reading responses were recorded on audiotape for later reading time measurement by stopwatch. In the memory task, the participants heard lists of pseudowords at a rate of one item per sec from audiotape and were asked to recall them immediately. Responses were recorded on minidisk to make offline checking possible. Testing started at List Length 3 and was continued until none of the lists at a certain length could be correctly recalled. We analyzed the number of lists re- called at Lengths 3 and 4 , since these had previously been found to be the ones that best discriminated between stimuli of varying phonological complexity. A within-subjects design was used so that all participants had to recall all four types of stimuli. The order of testing for the different types of pseudowords was permutated between participants so that lists of each type of stimulus were presented in all four positions. All reported within-subjects $p$ values have been corrected using the Greenhouse-Geisser method.

\section{Results}

Reading time. The time it took participants to read aloud the 12 stimuli of each kind varied according to pseudoword type $[F(3,42)=11.32, p<.0005]$. Mean reading time was $5.2 \mathrm{sec}(S D=1.4)$ for the list of short stimuli, 7.2 $\mathrm{sec}(S D=3.0)$ for the long-middle-redundantstimuli, $7.0 \mathrm{sec}(S D=2.3)$ for the long-end-redundant stimuli, and $7.6 \mathrm{sec}(S D=3.2)$ for the long-nonredundant stimuli. Planned contrasts showed that short pseudowords were read significantly faster than long-middle-redundant $(p<.001)$, long-end-redundant $(p<.005)$, or longnonredundant $(p<.0005)$ pseudowords, whereas there were no significant differences in reading time between the three kinds of long stimuli.

Recalled lists. The memory performance in terms of number of recalled lists of Lengths 3 and 4 can be seen in Figure 1. Both list lengths were subjected to one-way analyses of variance (ANOVAs). The results were similar for the two analyses. There was a highly significant effect of pseudoword type for three-item lists $[F(3,42)=15.95$, $p<.0001]$. Planned contrasts showed that fewer lists

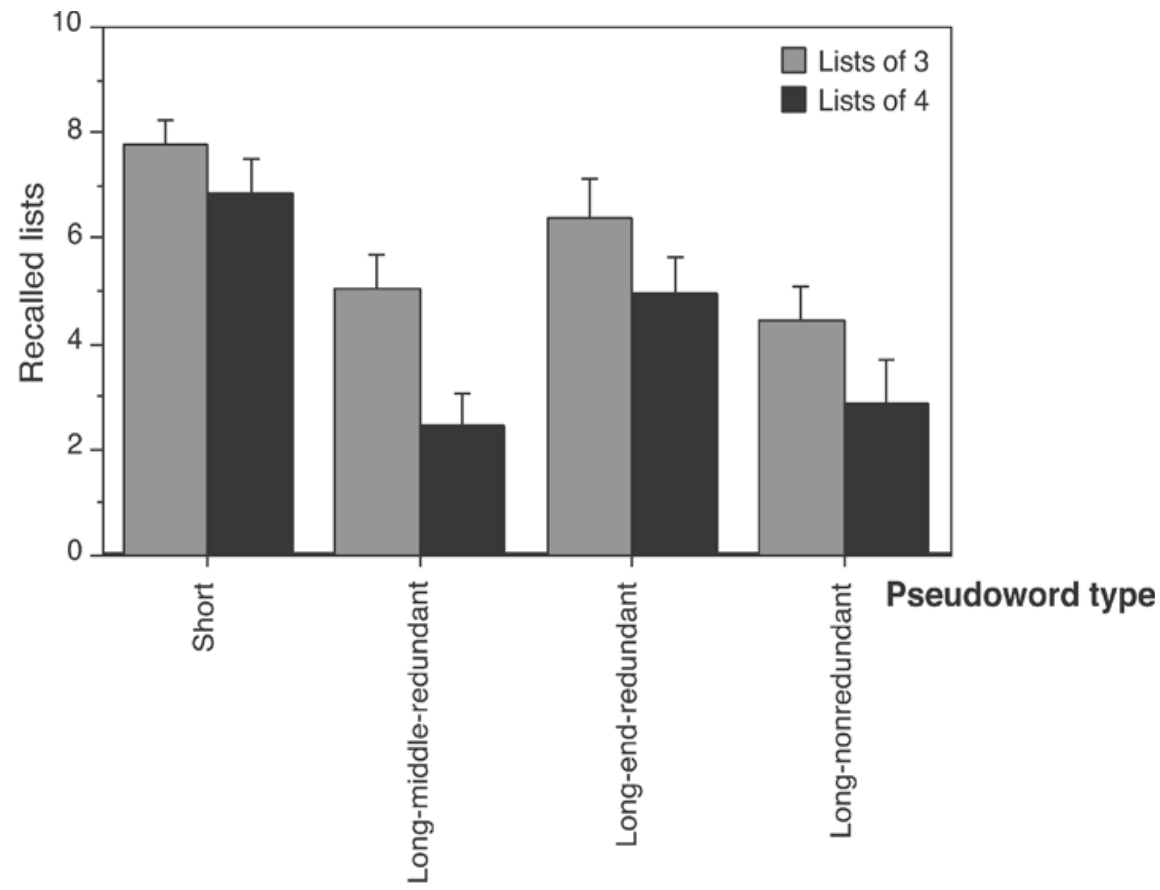

Figure 1. Recall of lists of three and four items in Experiment 1. The error bars denote standard errors of the mean. 
were recalled consisting of any of the three types of long stimuli relative to the short stimuli (all $p \mathrm{~s}<.05$ ). However, there was also a difference between the long-endredundant and the long nonredundant lists, favoring recall of end-redundant lists $(p<.005)$, whereas no significant difference was found between long-middle-redundantand long-nonredundant pseudowords. The same pattern of results was replicated for the lists consisting of four items $[F(3,42)=2,953, p<.0001]$. Short items were again recalled better than any of the three types of long items (all $p$ s <.005). However, lists with long-end-redundant items were also recalled better than lists with long-nonredundant items $(p<.001)$. There was no statistically reliable difference between lists of long-middle-redundant and long-nonredundant items $(p=.4236)$.

\section{Discussion}

Experiment 1 set out to test the possibility that redundancy in the phonological structure of pseudowords could boost participants' recall in immediate memory experiments with lists made up from limited pools of items. The results suggested that this was the case only when the redundancy occurred in a salient part of the pseudoword, like the end, where it could be easily detected by the participants. Redundancy in the middle of the pseudoword did not seem to affect memory performance relative to nonredundant pseudowords of equal phonological complexity. This pattern of results suggested that phonological redundancy helps memory only in special sections of a pseudoword, perhaps when it can aid more or less conscious memory strategies.

There is an alternative interpretation of the data, however. Added phonological redundancy is necessarily accompanied by added phonological similarity between list items. Phonological similarity is known to harm rather than help memory (Conrad \& Hull, 1964). The added phonological similarity could counteract the possible beneficial effects of phonological predictability to the extent that the effect is totally abolished when the redundancy occurs in the middle of the pseudoword and is diminished when it occurs at the end of the pseudoword. Experiment 2 was carried out to explore the effects of phonological predictability, trying to minimize possible phonological similarity effects.

\section{EXPERIMENT 2}

Three categories of pseudowords were studied in Experiment 2: short, long-end-predictable, and long-nonredundant. The short and the long-nonredundantstimuli were the same as in Experiment 1. The long-end-predictable pseudowords resembled the long-end-redundant pseudowords in Experiment 1. However, instead of the last syllable being shared between all stimuli, only its consonant was shared, whereas the vowel was always the same as the vowel in the middle syllable. Thus, the last syllable was perfectly predictable in these stimuli, if the middle syllable was known, but was different in different stimuli. If phonological similarity had affected the results in Experiment 1 , this effect should now be reduced and we might see improved recall of the long-end-predictable pseudowords relative to the short stimuli and the long-endredundant stimuli in that experiment.

\section{Method}

Participants. Fifteen students participated in this experiment, the majority for course credit. There were 5 males and 10 females (mean age $=24.0$ years, $S D=4.4$ years). None of the participants had been tested in Experiment 1. All were Finnish speakers with no known problems with reading or writing.

Stimuli. The short and long-nonredundant stimuli from Experiment 1 were used as stimuli. New long-end-predictable stimuli were made from the long-end-redundant stimuli in Experiment 1 by replacing the last vowel /e/ by the vowel in the middle syllable. This resulted in a perfectly predictable last syllable in these pseudowords.

Procedure. The procedure was the same as in Experiment 1 with the difference that there were only three conditions: short, longend-predictable, and long-nonredundant.

\section{Results}

Reading time. Reading time for the three lists of 12 stimuli was subjected to a one-way repeated measures ANOVA. The main effect of pseudoword type was highly significant $[F(2,28)=37.63, p<.0001]$. Planned contrasts showed that short pseudowords were read faster than both types of long pseudowords ( $p<.0001$ in both cases) and that long-end-predictable pseudowords were read faster than long-nonredundant pseudowords $($ means $=6.8$ sec, $S D=1.2$, and $7.3 \mathrm{sec}, S D=1.4$, respectively, $p<.05$ ). The latter difference was probably attributable to easier speech planning for the repeated vowel in the second and third syllables of the long-end-predictable stimuli. Its magnitude (7.4\%) is clearly smaller than that of differences that have been reported in the literature to produce word-length effects (Baddeley \& Andrade, 1994).

Recalled lists. Recall performance for lists of three and four items is shown in Figure 2. A repeated measures ANOVA on recall scores for lists of three pseudowords showed that the effect of pseudoword type was significant $[F(2,28)=11.64, p<.0005]$. The short pseudowords were recalled more often than the long-end-predictable $(p<$ $.05)$ and the long-nonredundant $(p<.0001)$ pseudowords. The difference between long-end-predictable and longnonredundant pseudowords approached significance $(p=$ .0718 ), reflecting the fact that performance for the longend-predictable lists was somewhat better.

A similar analysis on data for lists of four items also showed a significant main effect of pseudoword type $[F(2,28)=28.90, p<.0001]$. Short pseudowords were recalled better than long-end-predictable $(p<.0005)$ and long-nonredundant $(p<.0001)$ pseudowords. The advantage for long-end-predictablerelative to long-nonredundant items also reached significance in this comparison $(p<$ $.005)$.

To find out whether the reduction in phonological similarity between the long-end-redundantcondition of Experiment 1 and the long-end-predictable condition of Ex- 


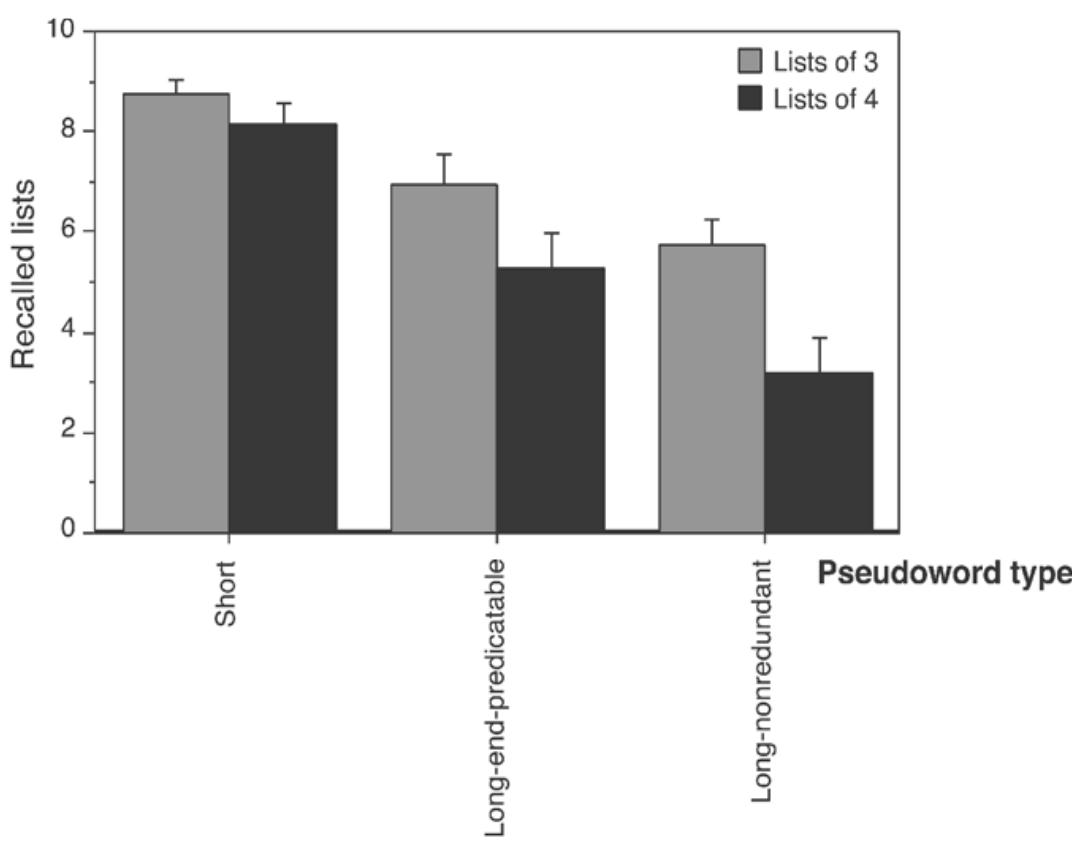

Figure 2. Recall of lists of three and four items in Experiment 2. The error bars denote standard errors of the mean.

periment 2 had made any difference to recall, two further ANOVAs, combining data from the two experiments, were performed. The long-middle-redundantcondition was left out of the data in Experiment 1 and the two conditions with end manipulations were considered equivalent. Mixed ANOVAs with experiment as a between-subjects variable and pseudoword type as a within-subjects variable were run on list scores for lists of three and four items. Neither showed a main effect of experiment ( $p$ values = .1519 and .4459 for lists of three and four, respectively). Nor were there statistically reliable interactions between the effects of experiment and pseudoword type ( $p$ values $=.6628$ and .4554 , for lists of three and four, respectively). The list memory data thus failed to show any effect of phonological similarity differences between the end-manipulated items in the two experiments.

\section{Discussion}

The second experiment was performed in order to try to reduce phonological similarity because that could have had a negative effect on recall of the end- and middle-redundant items in Experiment 1. Decreased phonologicalsimilarity between items, with only a shared consonant in the last $\mathrm{CV}$ syllable combined with a distinctive vowel in this experiment (as opposed to a whole shared CV syllable in Experiment 1), did not change the quantitative pattern of results in any way. There was still a great advantage for short pseudowords over pseudowords with a predictable end, and a somewhat smaller advantage for the long endpredictable items relative to the long nonredundant items. A direct comparison between experiments re- vealed no support for the hypothesis that phonological similarity between items with one shared syllable might have reduced recall performance in Experiment 1.

\section{EXPERIMENT 3}

The first two experiments manipulated the middle and end parts of pseudowords. However, previous work on phonological similarity effects for lists of real words has often used mixed lists of one-syllable rhyming words (e.g., mat, rat, cat) and words beginning with the same phonemes (e.g., mat, map; rat, rap). The third experiment contrasted redundancy at the beginnings of words with redundancy at the ends of words. The tip-of-the-tongue phenomenon (Brown \& McNeill, 1966; Caramazza \& Miozzo, 1997) and results of a false memory paradigm (Westbury et al., 2002) both suggest that the beginnings of words may have a special status in accessing phonological forms in long-term memory. Cuing by the initial sounds in a word is also a standard way of aiding word retrieval in patients with naming difficulties. Although the significance of the within-word position of shared phonological information has not been systematically investigated in immediate recall, it was hypothesized that pseudowords with redundant beginnings would be recalled less well than pseudowords with redundant ends. It was thought that the pool of pseudoword items repeated over lists would give rise to an activated set of representations. Phonological similarity effects would arise from competition between lexical (in this case pseudolexical) candidates belonging to such a set. If this set was ac- 
cessed in a left-to-right manner, shared first syllables could result in maximal confusion between items.

\section{Method}

Participants. Fifteen participants (mean age $=30.9$ years, range $=$ $22-51,8$ females) received course credit for taking part in the experiment, in connection with an open university psychology course.

Stimuli. Four sets of stimuli were used: the short, long-endredundant, and long-nonredundant items from Experiment 1 together with a pool of 12 long-beginning-redundant pseudowords sharing the initial syllable /ne/. This pool was created from the short stimuli in Experiment 1 by adding /ne/ to the beginning of the pseudowords.

Procedure. The procedure was as in Experiments 1 and 2.

\section{Results}

Reading time. The mean reading time was $5.7 \mathrm{sec}$ $(S D=1.8)$ for the 12 short stimuli, $7.6 \mathrm{sec}(S D=2.5)$ for the 12 long-end-redundant stimuli, $8.7 \mathrm{sec}(S D=2.5)$ for the 12 long-nonredundant stimuli, and $8.3 \mathrm{sec}(S D=2.4)$ for the 12 long-beginning-redundant stimuli. A one-way ANOVA showed a significant effect of pseudoword type $[F(3,42)=24.68, p<.0001]$. Planned contrasts showed that the short words took less time to read than did any of the other types of pseudowords did $(p<.0005$ in all cases). The only other significant comparison showed that long-end-redundant pseudowords were for the present participants faster to read than long-nonredundant pseudowords $(p<.05)$. This difference was approximately $13.5 \%$ of the shorter reading time.

Recalled lists. The number of recalled lists can be seen in Figure 3. There was a significant effect of pseudoword type $[F(3,42)=30.23, p<.0001]$ in lists of three items. Single-degree-of-freedom contrasts revealed that lists of short words were recalled more often than lists of any of the long pseudoword types ( $p<.0005$ in all cases). There was also a detrimental effect of redundant pseudoword beginning relative to redundant ending $(p<.0001)$ and a lack of redundancy $(p<.001)$ in the long pseudowords. The advantage for long-end-redundant relative to longnonredundant stimulus lists did not reach significance $(p=.1195)$.

The main effect of pseudoword type was also significant in the analyses of lists of four items $[F(3,42)=48.86, p<$ $.0001]$. The lists of short items were again recalled best $(p<.001$ in all comparisons). However, all other pairwise contrasts were also significant. Lists with items sharing the first syllable were harder to recall than were lists with long-end-redundant $(p<.0001)$ or long-nonredundant $(p<.05)$ stimuli. In these longer lists, the advantage for long-end-redundant lists relative to long-nonredundant lists was also highly significant $(p<.0001)$. Thus, the topdown support from redundant end syllables found in Experiment 1 was also replicated in this experiment.

\section{Discussion}

Experiment 3 was carried out to explore the effects of a shared initial syllable in immediate recall of pseudoword lists. Unlike the memory support provided by a redundant or predictable end syllable in Experiments 1 and 2, and the lack of a detectable effect of a shared middle syllable in Experiment 1, a shared initial syllable significantly harmed recall, producing the well-known phonological similarity effect. Shared initial syllables were suspected to produce effects different from those of other shared parts of the pseudowords on the basis of a hypothesis that phonological similarity effects in general are caused by competition among phonologically similar lexical items. In the case of many word stimuli on a list sharing some

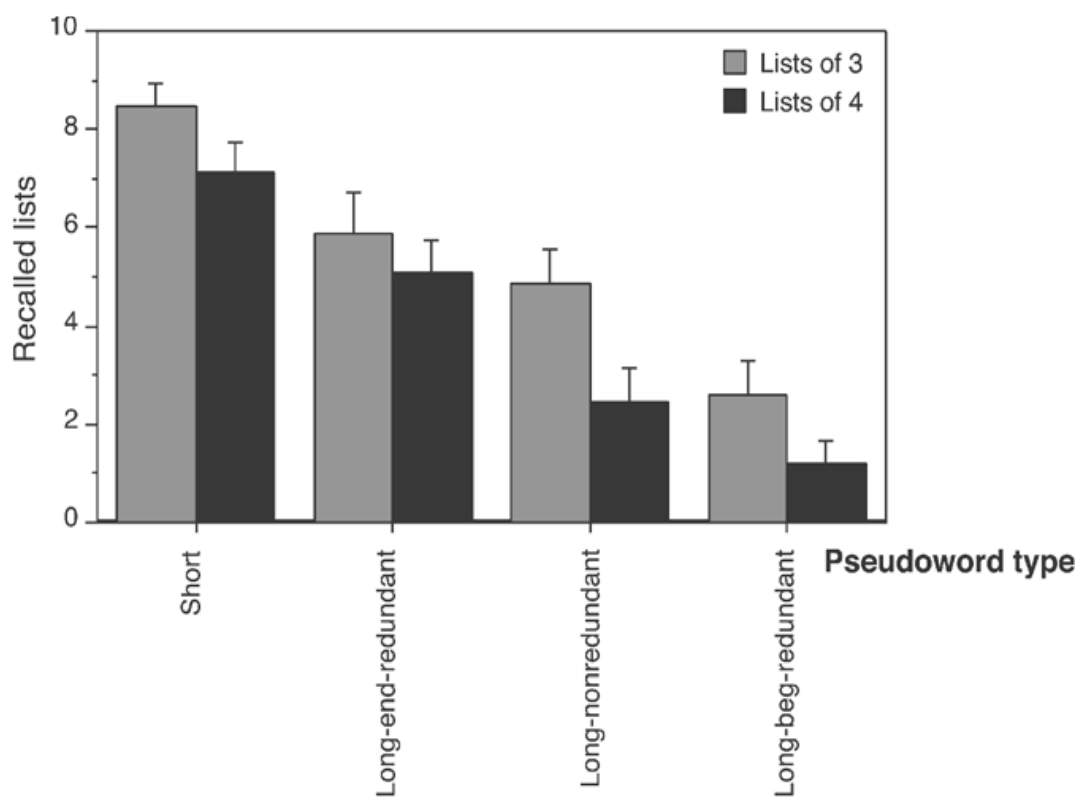

Figure 3. Recall of lists of three and four items in Experiment 3. The error bars denote standard errors of the mean. 
phonological sequences, this could lead to mutual inhibition among the set of activated lexical entries that are used to reconstruct phonologically incomplete memory traces. Confusion following competition would be especially pronounced for words that share their beginnings if word onsets play a special role in access to the phonological lexicon. This is suggested by three phenomena in word access: tip-of-the-tongue data (Brown \& McNeill, 1966), aphasic naming of pictures that share the CV beginnings of their names (Laine \& Martin, 1996), and false recognition of phonological lures in the context of phonologically similar lists (Westbury et al., 2002). Pseudowords are potential words but lack preexisting representations in long-term memory. The redintegration process for them would therefore have to depend on representations built up during the experiment. Lack of established long-term memory entries and semantic anchors could make them vulnerable to on-line intraexperimental competition effects (e.g., proactive inhibition) and could therefore accentuate phonological similarity effects. The reason for hypothesizing a special role for the beginnings of pseudowords is that these items would be treated as novel words and therefore be coded in the same way as would real words, with the beginning given more weight in access.

The results so far have been based on the more conventional tradition of scoring memory for whole items. However, when nonword stimuli are used it is also possible to investigate memory at a more fine grained phonological level. Memory on the phonological level is hard to study with lists consisting of real words, since the majority of items are typically either completely recalled (e.g., 76\% in the study by Gathercole et al., 1999) or completely phonologically incorrect (e.g., 13\% in the study by Gathercole et al., 1999). Partial errors, in which some of the phonemes of a word are recalled correctly, are rare ( $12 \%$ in the study by Gathercole and her colleagues). In contrast, partially recalled pseudowords have been observed to be more common in immediate list recall $(46 \%$ in the data of Gathercole et al., 1999). The availability of lexical representations for real words and the unwillingness of participants to respond with nonwords in a list of words makes it more difficult to study phonological forgetting in the context of real words. In the present experiment, an error analysis made it possible to study the effects of top-down beneficial and harmful influences at the syllable level.

\section{ERROR ANALYSIS OF EXPERIMENTS 1-3}

In order to try to disentangle the possibly counteractive effects of phonological similarity and phonological redundancy and separate effects on different syllable positions, an error analysis was carried out on the data from Experiments 1-3. We expected to see two effects: a helpful effect on the recall of the redundant syllables and a harmful effect on all other syllables.

\section{Method}

First, a series of ANOVAs was run to establish that the respective error rates for short items and long-nonredundant items, shared by the three experiments, did not significantly differ between experiments. These analyses confirmed that the overall error rates in the three experiments were similar. After that, data for each class of stimuli were pooled from the three experiments and proportions correct were calculated for all presented stimuli both at the item level and at the level of first syllables, middle syllables, and last syllables, separately. The proportions correct for four different types of threesyllable phonologically redundant stimuli-beginning-redundant, middle-redundant, end-redundant, and end-predictable - were compared with the baseline proportion correct for nonredundant stimuli.

\section{Results and Discussion}

The percentages of correctly recalled items and syllables are shown in Table 1. Statistical comparisons were carried out on these data. This is conservative in the sense that it possibly underestimates differences between stimulus types. This is because all syllables in omitted pseudowords (15.54\% of all errors) are considered equally forgotten. The proportion of omissions of all errors was $14.06 \%$ for nonredundant pseudowords, $9.4 \%$ for beginning-redundant pseudowords, $25 \%$ for middle-redundant pseudowords, $16.94 \%$ for end-redundant pseudowords, and $12.89 \%$ for end-predictable pseudowords. Since omissions are overrepresented for the middle-redundant pseudowords, the picture for these stimuli is somewhat less clear than for the other types.

The distributions of correct and incorrect responses for all three syllables were highly significantly different over the different stimulus types $\left[\chi^{2}(4)=51.43, p<.0001\right.$, for the first syllable; $\chi^{2}(4)=136.51, p<.0001$, for the second syllable; and $\chi^{2}(4)=401.05, p<.0001$, for the last syllable]. Pairwise comparisons were made of data for each syllable position and for each redundant stimulus type with the corresponding syllable position for nonredundant stimuli. In addition, data for end-redundant stimuli were compared with data for end-predictable

Table 1

Proportion of Correct Responses in Experiments 1-3

\begin{tabular}{lccccc}
\hline Stimulus Type & $\begin{array}{c}\text { Number of } \\
\text { Stimuli }\end{array}$ & $\begin{array}{c}\text { Correct Items } \\
(\%)\end{array}$ & $\begin{array}{c}\text { Correct First } \\
\text { Syllable (\%) }\end{array}$ & $\begin{array}{c}\text { Correct Middle } \\
\text { Syllable (\%) }\end{array}$ & $\begin{array}{c}\text { Correct Last } \\
\text { Syllable (\%) }\end{array}$ \\
\hline Nonredundant & 3,220 & 70.84 & 89.60 & 80.68 & 79.04 \\
Beg-redundant & 640 & 58.44 & 93.75 & 67.81 & 66.56 \\
Mid-redundant & 960 & 69.58 & 87.19 & 89.06 & 71.88 \\
End-redundant & 2,700 & 75.3 & 86.59 & 78.89 & 91.89 \\
End-predictable & 1,300 & 80.31 & 92.46 & 85.46 & 87.38 \\
\hline
\end{tabular}


Table 2

Pairwise Comparisons Between Syllable Recall of

Phonologically Redundant and Nonredundant Items

\begin{tabular}{|c|c|c|c|}
\hline Comparison & $\chi^{2}(1)$ & $p<$ & Tested Effect \\
\hline \multicolumn{4}{|l|}{ First Syllable } \\
\hline Nonredundant vs. beg-redundant & 10.50 & .005 & Top-down beneficial \\
\hline Nonredundant vs. mid-redundant & 4.40 & .05 & Phon. sim. harmful \\
\hline Nonredundant vs. end-redundant & 12.76 & .0005 & Phon. sim. harmful \\
\hline Nonredundant vs. end-predictable & 8.78 & $\begin{array}{c}.005 \\
\text { (wrong way) }\end{array}$ & Phon. sim. harmful \\
\hline \multicolumn{4}{|l|}{ Middle Syllable } \\
\hline Nonredundant vs. beg-redundant & 52.49 & .0001 & Phon. sim. harmful \\
\hline Nonredundant vs. mid-redundant & 36.14 & .0001 & Top-down beneficial \\
\hline Nonredundant vs. end-redundant & 2.94 & n.s. & Phon. sim. harmful \\
\hline Nonredundant vs. end-predictable & 14.36 & $\begin{array}{c}.0002 \\
\text { (wrong way) }\end{array}$ & Phon. sim. harmful \\
\hline \multicolumn{4}{|l|}{ Last Syllable } \\
\hline Nonredundant vs. beg-redundant & 46.86 & .0001 & Phon. sim. harmful \\
\hline Nonredundant vs. mid-redundant & 21.68 & .0001 & Phon. sim. harmful \\
\hline Nonredundant vs. end-redundant & 189.90 & .0001 & Top-down beneficial \\
\hline Nonredundant vs. end-predictable & 42.69 & .0001 & Top-down beneficial \\
\hline End-redundant vs. end-predictable & 20.56 & .0001 & Size of phon. sim. \\
\hline
\end{tabular}

Note-“Top-down" refers to beneficial redundancy effects and "Phon. sim." to harmful similarity effects.

stimuli in order to estimate the impact of the greater phonological similarity of the end-redundant stimuli. The results of these comparisons are shown in Table 2. All four relevant comparisons were compatible with the hypothesis that phonological redundancy has a positive topdown effect on recall of the redundant syllables. The second hypothesis, stating that phonological similarity harms the recall of all other syllables but the one that is shared between stimuli, got support from five significant comparisons. One additional effect approached significance, showing a trend for better recall of the middle syllable in nonredundant than in end-redundant stimuli $\left[\chi^{2}(1)=\right.$ 2.94, $p=.0864]$. The remaining two results are in the opposite direction. Both involved the end-predictable stimuli and suggested that there was no harmful phonological similarity effect for these stimuli. Instead, recall was enhanced for all three syllables.

Interestingly, the seeming lack of effect of a redundant middle syllable in Experiment 1 seems to have stemmed from the opposing effects of memory enhancement for the middle syllable and memory impairment for the two other syllables, the two effects canceling each other out. It is also clear that the impact of the two opposing forces varied as a function of syllable position. This should be seen against the pattern of recall in the nonredundant baseline condition. In this condition, the first syllable was better remembered than the two other syllables $\left[\chi^{2}(1)=101.09\right.$, $p<.0001$, and $\chi^{2}(1)=135.75, p<.0001$, for the middle and last syllables, respectively]. Recall of the two later syllables did not differ. Phonological similarity was clearly most harmful when it affected the first syllable of the items. Unlike in the case of the middle-redundant stimuli, the beneficial effect of redundancy was not here able to match the detrimental similarity effect of a shared first syllable impairing recall of both later syllables. This sug- gests that the first syllable carried more weight than did the other syllables as an item identifier, and that this role could not be taken over by the other two syllables when the first syllable was deprived of its ability to differentiate between stimulus items. It should be noted that the remaining portions of the stimuli were the same as the wellrecalled short (two-syllable) stimuli.

The results also revealed that repetition (but not predictability) at the ends of the pseudowords harmed recall of the first syllables, and somewhat less clearly recall of the middle syllables. Still, overall, phonological redundancy at the ends of pseudowords appeared to be more helpful than harmful, suggesting that the top-down beneficial effect was greater than the harmful phonological similarity effect when no phonological material followed the redundancy. The whole pattern of results suggests that the harmful effect of phonological similarity is stronger in the forward than in the backward direction within items.

The majority (79.88\%) of errors in recall were phonological item errors, the remaining errors being omissions $(15.54 \%)$ and order errors (4.59\%). Of the phonological item errors, only a small fraction represented intrusions from other lists (2.19\% of all errors). Figure 4 shows the proportions of correctly recalled first, middle, and end syllables in the phonologically incorrect responses. Note that such data may slightly misrepresent differences between item types by excluding forgetting resulting in omissions or order errors. The proportions show very clearly that redundant syllables were boosted relative to the baseline condition of nonredundant stimuli in these incorrect responses. For the beginning-redundant stimuli, the first syllable was recalled correctly in $99.56 \%$ of cases - that is, in all but 1 of a total of 227 responses. Recall of the middle syllable was boosted from the $41.12 \%$ of the nonredundant stimuli to $87.79 \%$ for the middle-redundant 


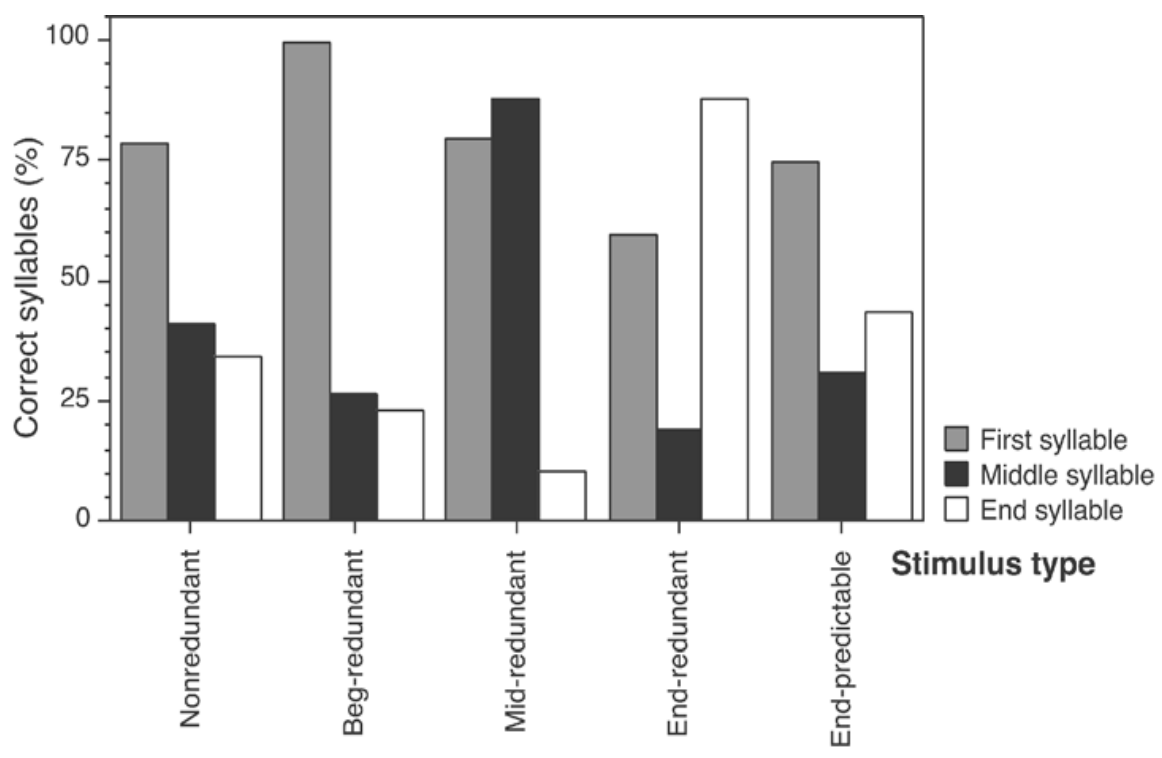

Figure 4. Proportion of correctly recalled syllables in incorrectly recalled items.

pseudowords, and recall of the final syllable was helped from $34.22 \%$ for the nonredundant items to $87.84 \%$ for the end-redundant stimuli in responses with phonological errors. Clearly, phonological redundancy protected the redundant syllable from error, although the effect on wholeitem level could even be the opposite. It looks as if this effect depended on the set of activated syllables in memory. In the case of end-redundant stimuli, 12 pseudowords sharing the same third syllable were more or less available to repair a degraded last syllable. Choice of any one of them or an added effect of last syllables would have resulted in successful repair. In the case of end-predictable stimuli, a set of seven different end-syllables could have been activated in the experimental condition. In incorrect responses, the last syllable was correct in only $43.4 \%$ of the cases, showing a much smaller effect than in the endredundant stimuli despite a very similar outcome on wholeitem level. The memory outcome could be related to the finding by Roodenrys and his colleagues (Roodenrys, Hulme, Lethbridge, Hinton, \& Nimmo, in press) that words from larger phonological neighborhoods and words from high-frequency neighborhoods are better remembered than words from small and low-frequency neighborhoods. The net effect of all the phonological activations would help to support forgotten phonemes. It appears that immediate recall of both words and pseudowords involves processes that benefit from the phonological similarity of activated items. These processes seem to mediate top-down repairing influences from longterm memory. Other aspects of these processes may be responsible for the harmful effects of phonological similarity, observed when items in a list share sounds. These would result in difficulties with keeping track of item identity (important, e.g., for memory for item order). The first syllable could have a special role in identifying the item.

\section{GENERAL DISCUSSION}

We explored the effect of phonological redundancy on immediate recall in three experiments. We systematically examined shared first-, middle-, and last-syllable effects on immediate serial recall of trisyllabic pseudowords. Our results showed a pronounced harmful phonological similarity effect on recall when the stimuli shared the first syllable. No effect on item recall was seen when the middle syllable was shared, and recall was better when the last syllable was shared or could be predicted from context. These results were, however, qualified by findings of syllable-level effects in an error analysis. We also asked whether redintegration, or similar top-down influences, could explain the lack of (or highly reduced) item-length effect for Finnish-sounding pseudowords that differed in pronunciation duration but not in other aspects of phonological quality. In no condition were we able to help memory for three-syllable items to the level of two-syllable stimuli. We therefore think it unlikely that the previously reported lack of item-length effect in Finnish (Service, 1998) could have resulted exclusively from beneficial topdown effects counteracting time-based decay.

A comparison of middle-redundant and nonredundant lists first suggested that there was no phonological similarity effect for this type of redundancy. Recall for totally nonredundant three-syllable pseudowords was equally good as for items with a shared middle syllable. However, the error analysis suggested that beneficial and harmful effects balanced each other out when the middle syllable was redundant. Similarly, no statistically reliable improvement in recall took place for items with a predictable last syllable that shared only its consonant with the other list items (Experiment 2) relative to items that had a shared $\mathrm{CV}$ combination as the last syllable (Experiment 1 ). Thus, 
the reduced phonological similarity of the end-predictable items first did not seem to have any detectable effect on recall in this case. Again, the error analysis suggests that there was a balancing out of a small harmful phonological similarity effect affecting the first two syllables in the end-redundant stimuli, and a clear boost of recall of the last syllable in these items, in contrast to a small, even, enhancement of all syllables of the end-predictable stimuli. The latter effect is different from the localized effects of syllable enhancement and degradation seen in the conditions with repeated syllables. It suggests that the endpredictable items were in general easier than the other types of items. This could be because they were faster to say, in line with a time-decay hypothesis. There are also two explanations that would be compatible with a phonological complexity hypothesis of phonologicalloop capacity. Items with repeated vowels in consecutive syllables could be easier from the point of view of representations at an abstract level, which are needed in storage and rehearsal, because the representation of a repeated vowel in a vowel frame would be simpler than a representation of two different vowels in a vowel frame (see Service, 1998). The difference could also be at the level of the speech planning system, since the planning of speech gestures would be easier when vowel features remain the same over two syllables. The output speech planning explanation is supported by recent data showing that lists with easier articulatory transitions between items are remembered better than lists with greater differences in articulatory features between the ends and beginnings of adjacent items (Murray \& Jones, in press).

It is not possible to determine whether the improvement in recall seen for the end-manipulated items depended on conscious or unconscious strategies. Conscious strategies could have involved rehearsal without the last syllable or the guessing of deteriorated last syllables at the recall stage. The error pattern, showing signs of the harmful presence of phonological similarity, does not support the assumption of truncated rehearsal. It is, however, not possible to totally refute this possibility if truncation as an operation results in some resource consumption and this effect, or the employment of the strategy, depends on syllable position of the redundant material. We find truncated rehearsal counterintuitive but are currently pursuing this possibility in an experiment with explicit instructions to truncate. The superior memory for redundant relative to predictable last syllables is not in line with another conscious strategy, that of guessing. Instead, subconscious effects could have resulted in improved restoration or redintegration (Brown \& Hulme, 1995; Hulme, Newton, Cowan, Stuart, \& Brown, 1999; Schweickert, 1993) of imperfectly encoded or partly degraded representations based on multiple representations of the correct final syllable in the set of activated stimulus items.

An intriguing finding was the discrepancy between redundancy effects in different parts of the stimuli. It should immediately be noted that we do not know the extent to which the exact pattern of effects generalizes to stimuli with different phonotactic structures. It could well be, for instance, that lexical sets of monosyllabic words are treated differently than sets with multisyllabic or mixed items. However, it is interesting to note that top-down phonotactic regularity effects also helped the end part (VC) of CVC items in the study by Gathercole and her colleagues (Gathercole et al., 1999). The net effect of phonological similarity in our study seemed to depend on the balance between helpful and harmful syllable-level effects. Sometimes there was no effect at the level of item recall, as in the case of the shared middle syllables, or beneficial top-down effects dominated, as in the case of predictable or shared end syllables. Such an interaction between syllable position and phonological redundancy could have resulted from lexical influences if the shared syllable/ne/ occured with different frequencies in different syllable positions in Finnish words. However, the frequency of /ne/ as initial syllable in Finnish words is 164 in 201,000 words (Sadeniemi, 1990) and as word-final syllable is 151 in 207,256 words (Tuomi, 1980) (the latter dictionary is based on the former but also includes words from its footnotes). Thus, there is practically no difference in the lexical frequency of /ne/ as word-initial relative to word-final syllable.

It should be noted that the role of different syllable positions may be linked to prosodic characteristics of the stimuli and that it could vary between languages. Longoni, Richardson, and Aiello (1993) have reported a harmful phonological similarity effect for four-syllable Italian words that share four sounds in the middle of the word. However, unlike words in standard Finnish, which always have the main stress on the first syllable, these words had the main stresses in the shared middle parts of the words.

Li, Schweickert, and Gandour (2000) contrasted two hypotheses of the detrimental effect of phonological similarity. According to the "acid bath" hypothesis (Posner, 1966), incoming phonologically similar items interfere with the memory traces of earlier presented items with noisier memory representations as a result (e.g., the same phonemes activated by multiple items). The alternative hypothesis suggests that similar items make it more difficult to guess partly degraded representations at the time of recall since the degraded traces may not have enough distinctive information left to separate them from other items on the list. This could create order errors, especially for short words, when, for instance, map had deteriorated to *ap and rap, map, and sap were available to repair it. In the case of nonwords, partially correct responses could occur if parts of the stimuli (e.g., phonemes or syllables) could be individually repaired and there would be no lexicality monitor to reject nonwords as responses. The results of Li et al. were not clear enough to reject either the acid bath or the impaired guessing hypothesis, but the authors concluded that they were explained somewhat better by assuming impairment at the reconstruction stage. Their data also showed that shared phonemes in any position (e.g., spit, tips) sufficed to create a phonological similarity effect, but that shared phonemes in the same positions in the word (e.g., spit, spot) accentuated the effect. The present experiments suggest the hypothesis that specific 
parts of the items (here the first syllables) could play a dominating role in restricting the set of items activated in the redintegration process. When first syllables were shared, perhaps more items were allowed to compete to repair lost information. For the redundant syllables, this would have been helpful, and for the rest it would have created harmful interference. In general, data on item errors in pseudoword lists could be more informative about phonological forgetting and repair than data about real words since strong lexical representations are not available. When real words are studied, paradigms allowing maximal proactive inhibition (see, e.g., Fallon, Groves, \& Tehan, 1999) may be better suited than simple list recall for studying effects on the phonological level. The data from our study with pseudowords point to the need for models of phonological memory to represent sublexical structure of wordlike stimuli, including syllable structure, phoneme number and order, $\mathrm{C} / \mathrm{V}$ status, and so on (Hartley \& Houghton, 1996; Service, 1998).

The present data showed impaired recall of nonboosted syllables that was more pronounced for syllables following the shared syllable, whereas the first syllable showed relative immunity to recall errors. The results suggest that the phonological similarity effect may be a result of two processes: lexical (or pseudolexical) competition that arises from the way item identity is coded in the phonological store together with repair processes affecting degraded traces that have a variable probability of success. In Finnish, the stressed first syllable of the word appears to have a special status as an identifier. Recent evidence from a false recognition paradigm with English CVC words suggests that shared word beginnings (in this case $\mathrm{Cs}$ or CVs) are more deceptive than shared word endings (in this case VCs) (Westbury et al., 2002). It would be economical to assume that the phonological buffer uses similar identification cues as episodic word access. If the first syllable cannot be used as an identifier (e.g., /ne/ to distinguish between /nehiru/ and /nejalu/), the probability that a degraded trace (e.g., /ne**ru/) is repaired by another similar trace (e.g., /nejalu/) should increase. Even if the last syllable/ru/ should be enough to distinguish the item from all others, competition from other active middle syllables could defeat the correct one in a process with identification weights decreasing from left to right in an item. Many unknowns regarding phonotactic probabilities, relative importance of consonants and vowels, the units of processing (e.g., phonemes, syllables) need to be known for successful modeling of phonological item forgetting in short-term memory.

To summarize, we found both helpful and harmful effects of phonological redundancy on recall of lists of pseudowords stemming from a limited pool. Harmful effects dominated when the stressed beginning of the pseudoword was shared between items, whereas a helpful net effect was observed when the ends of the pseudowords were shared. A facilitatory effect of a qualitatively different kind was detected when the vowel in the last syllables of the pseudowords was shared with the vowel in the middle syllables. This effect could have resulted from simpler memory representations or an articulatory advantage in speech planning for these items. Both explanations would be in line with the hypothesis that phonological complexity acts as a memory load (Service, 1998).

\section{REFERENCES}

Baddeley, A. [D.] (1986). Working memory. Oxford: Oxford University Press, Clarendon Press.

BadDeley, A. [D.], \& ANDRADE, J. (1994). Reversing the word-length effect: A comment on Caplan, Rochon, and Waters. Quarterly Journal of Experimental Psychology, 47A, 1047-1054.

Baddeley, A. D., \& Hitch, G. (1974). Working memory. In G. H. Bower (Ed.), The psychology of learning and motivation (Vol. 8, pp. 47-89). New York: Academic Press.

Baddeley, A. D., Thomson, N., \& Buchanan, M. (1975). Word length and the structure of short-term memory. Journal of Verbal Learning \& Verbal Behavior, 14, 575-589.

Brown, G. D. A., \& Hulme, C. (1995). Modeling item length effects in memory span: No rehearsal needed? Journal of Memory \& Language, 34, 594-621.

Brown, R., \& McNeill, D. (1966). The "tip of the tongue" phenomenon. Journal of Verbal Learning \& Verbal Behavior, 5, 325-337.

Caplan, D., Rochon, E., \& Waters, G. (1992). Articulatory and phonological determinants of word length effects in span tasks. Quarterly Journal of Experimental Psychology, 45A, 177-192.

CaPlan, D., \& Waters, G. S. (1994). Articulatory length and phonological similarity in span tasks: A reply to Baddeley and Andrade. Quarterly Journal of Experimental Psychology, 47A, 1055-1062.

Caramazza, A., \& Miozzo, M. (1997). The relation between syntactic and phonological knowledge in lexical access: Evidence from the "tip-of-the-tongue" phenomenon. Cognition, 64, 309-343.

ConRad, R. \& Hull, A. J. (1964). Information, acoustic confusion and memory span. British Journal of Psychology, 55, 429-432.

Fallon, A. B., Groves, K., \& Tehan, G. (1999). Phonological similarity and trace degradation in the serial recall task: When CAT helps RAT but not MAN. International Journal of Psychology, 34, 301-307.

Gathercole, S. E., Frankish, C. R., Pickering, S. J., \& Peaker, S. [M.] (1999). Phonotactic influences on short-term memory. Journal of Experimental Psychology: Learning, Memory, \& Cognition, 25, 84-95.

Gathercole, S. E., Pickering, S. J., Hall, M., \& Peaker, S. M. (2001). Dissociable lexical and phonological influences on serial recognition and serial recall. Quarterly Journal of Experimental Psychology, 54A, 1-30.

Hartley, T., \& Houghton, G. (1996). A linguistically constrained model of short-term memory for nonwords. Journal of Memory \& Language, 35, 1-31.

Hulme, C., Maughan, S., \& Brown, G. D. (1991). Memory for familiar and unfamiliar words: Evidence for a long-term memory contribution to short-term memory span. Journal of Memory \& Language, 30, 685701.

Hulme, C., Newton, P., Cowan, N., Stuart, G., \& Brown, G. (1999). Think before you speak: Pauses, memory search, and trace redintegration processes in verbal memory span. Journal of Experimental Psychology: Learning, Memory, \& Cognition, 25, 447-463.

Knott, R., Patterson, K., \& Hodges, J. R. (2000). The role of speech production in auditory short-term memory: Evidence from progressive fluent aphasia. Neuropsychologia, 38, 125-142.

Laine, M., \& Martin, N. (1996). Lexical retrieval deficit in picture naming: Implications for word production models. Brain \& Language, 53, 283-314.

LI, X., Schweickert, R., \& Gandour, J. (2000). The phonological similarity effect in immediate recall: Positions of shared phonemes. Memory \& Cognition, 28, 1116-1125.

Longoni, A. M., Richardson, J. T. E., \& Aiello, A. (1993). Articulatory rehearsal and phonological storage in working memory. Memory \& Cognition, 21, 11-22.

Murray, A., \& Jones, D. M. (in press). No, Welsh digits are not shorter 
than English digits when spoken: Implications for the character of subvocal rehearsal. Journal of Experimental Psychology: Learning, Memory, \& Cognition.

NAIRnE, J. S. (1990). A feature model of immediate memory. Memory \& Cognition, 18, 251-269.

Posner, M. I. (1966). On the role of interference in short-term retention. Journal of Experimental Psychology, 72, 221-231.

Roodenrys, S., Hulme, C., Lethbridge, A., Hinton, M., \& Nimmo, L. M. (in press). Word frequency and phonological neighborhoodeffects on verbal short-term memory. Journal of Experimental Psychology: Learning, Memory, \& Cognition.

SADENIEMI, M. (1990). Nykysuomen sanakirja [Dictionary of modern Finnish] (18th ed., Vol. 2). Porvoo: Werner Söderström Osakeyhtiö.

SAInt-Aubin, J., \& PoIRIER, M. (2000). Immediate serial recall of words and nonwords: Tests of the retrieval-based hypothesis. Psychonomic Bulletin \& Review, 7, 332-340.

Schweickert, R. (1993). A multinomial processing tree model for degradation and redintegration in immediate recall. Memory \& Cognition, 21, 168-175.

Service, E. (1998). The effect of word-length on immediate serial recall depends on phonological complexity, not articulatory duration. Quarterly Journal of Experimental Psychology, 51A, 283-304.

TuомI, T. (1980). Suomen kielen käänteissanakirja [Reverse dictionary of modern standard Finnish] (2nd rev. ed.). Hämeenlinna: Finnish Literature Society.

Westbury, C., Buchanan, L., \& Brown, N. R. (2002). Sounds of the neighborhood:False memories and the structure of the phonological lexicon. Journal of Memory \& Language, 46, 622-651.

\section{APPENDIX}

Stimuli Used in Experiments 1-3

\begin{tabular}{cccccc}
\hline Pseudowords & Nonredundant & $\begin{array}{c}\text { Beginning- } \\
\text { Redundant }\end{array}$ & $\begin{array}{c}\text { Middle- } \\
\text { Redundant }\end{array}$ & $\begin{array}{c}\text { End- } \\
\text { Redundant }\end{array}$ & $\begin{array}{c}\text { End- } \\
\text { Predictable }\end{array}$ \\
\hline hali & haliso & nehali & haneli & haline & hilana \\
hiru & hiruma & nehiru & hineru & hirune & hirunu \\
jalu & jaluke & nejalu & janelu & jalune & jalunu \\
jomi & jomite & nejomi & jonemi & jomine & jomini \\
kula & kulani & nekula & kunela & kulane & kulana \\
kämö & kämörä & nekämö & känemö & kämöne & kämönö \\
mili & milisa & nemili & mineli & miline & malini \\
pame & pamelu & nepame & paneme & pamene & pamene \\
rovi & rovimo & nerovi & ronevi & rovine & rovini \\
sira & sirava & nesira & sinera & sirane & sirana \\
timä & timänö & netimä & tinemä & timäne & timänä \\
vuro & vuropi & nevuro & vunero & vurone & vurono \\
\hline
\end{tabular}

Note-Finnish orthography is close to phonetic script with ä pronounced as /æ/ and ö as /œ/.

(Manuscript received December 18, 2001;

revision accepted for publication July 8, 2002.) 